\title{
Low Voltage and Low Vacuum- When Worlds Collide
}

\author{
Bradley L. Thiel and Vasiliki Tileli \\ College of Nanoscale Science and Engineering, SUNY-Albany, Albany, NY 12203
}

Low voltage and low vacuum scanning electron microscopy have both been advocated for stable secondary electron (SE) imaging of uncoated insulating specimens. For the low voltage technique, the primary beam energy is chosen to be at or below the so-called E2 cross-over point, which is the incident energy for which the combined secondary and backscattered emission coefficients equal unity. Ideally, no net charge is deposited in the specimen. If the beam energy is chosen below E2, more electrons are emitted initially, causing the specimen to develop a net positive charge, which in turn suppresses some of the subsequent secondary electron emission until steady-state is achieved. Typical E2 values for insulating materials range from $3 \mathrm{keV}$ down.[1]

In the low vacuum approach, a small pressure of gas is maintained in the specimen chamber; typically ranging from 10 to $10,000 \mathrm{~Pa}$. electrons emitted from the specimen are accelerated by an applied electric field and initiate an ionization cascade in the gas. Positive gaseous ions drift to the specimen surface and remove excess electronic charge. This approach has the advantage of allowing the beam energy to be chosen based on other experimental constraints, such as electron optics or microanalysis considerations. However, early low vacuum instruments did not perform well at low voltages because of strong scattering of low energy electrons by the gas molecules.

The development of immersion lens low vacuum instruments also capable of operating at low voltages raises the question of what are the implications of working with a positively charging specimen (i.e., below E2) while positive ions are being generated in the cascade. To investigate this problem, images were taken of a lithographic mask consisting of a $6.25 \mathrm{~mm}$ thick sheet of silica with $80 \mathrm{~nm}$ thick chromium lines on its surface. The instrument used was an FEI Nova NanoSEM, equipped with an Everhart-Thornley SE detector for high vacuum operation and a Helix ${ }^{\mathrm{TM}}$ detector for low vacuum mode. $[2,3] \mathrm{By}$ first imaging in high vacuum, the E2 energy for this system was found to lie between 2.0 and $2.5 \mathrm{keV}$, as seen in figure 1. The mask was then imaged in low vacuum mode using $70 \mathrm{~Pa}$ of water vapor for the imaging gas. Stable, high contrast images free from electronic charging artifacts were obtained for $2.5 \mathrm{keV}$ primary beam energies and above, shown in figure 2. However, when the beam energy was lowered to $2.0 \mathrm{keV}$ SE contrast was lost abruptly, consistent with the onset of net positive charging.

The loss of SE signal can be explained by considering the electrostatic field structure that results working above and below the E2 point. The electrode structure, sample arrangement, and electrostatic field structure are illustrated in figure 3. Above the anode/detector, the field terminates on the grounded pressure limiting aperture. Below the anode plane the field terminates on the grounded ion trap, however it also lenses through the trap down to the next closest ground plane. As the mask is a dielectric, the field penetrates through down to the specimen stage. The surface of the specimen sits at a 
slightly positive potential in the field, as determined by the anode bias, mask thickness, and dielectric constant. Regardless, the surface is still at a lower potential than the anode, so secondary electrons are emitted and detected. When the beam energy exceeds E2, a flux of gaseous positive ions flows to the surface to mediate any excess negative charging. Thus, the system self-regulates to maintain a situation approximately given by figure 3a. Below E2, however, an additional field component results from the accumulation of positive charge (i.e. holes) in the secondary electron escape depth. Consequently, the electrostatic field structure is perturbed through the creation of a saddlepoint just above the mask surface, as shown in figure $3 \mathrm{~b}$. If the vertical component of an escaping electron's kinetic energy does not exceed the value of the saddlepoint, the electron is reflected back to the surface and not detected. Consequently, the SE signal is lost. In conclusion, while low voltage favors imaging insulators with beam energies less than E2, low vacuum favors imaging above E2.

[1] D. Joy, Database of Electron Solid Interactions, http://web.utk.edu/ srcutk/htm/interact.htm.

[2] M. Toth, et al., Appl. Phys. Lett. 88, 023105 (2006).

[3] B.L. Thiel, et al., Rev. Sci. Inst., in press (2006).

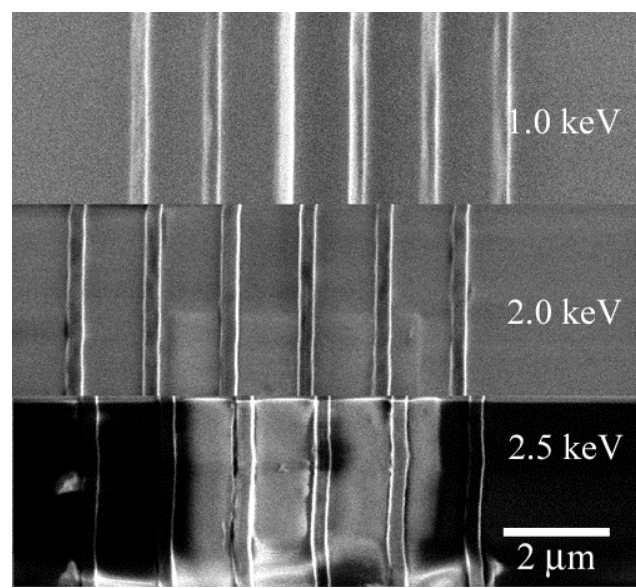

Figure 1. High vacuum images of mask at $1.0,2.0$, and $2.5 \mathrm{keV}$.

a

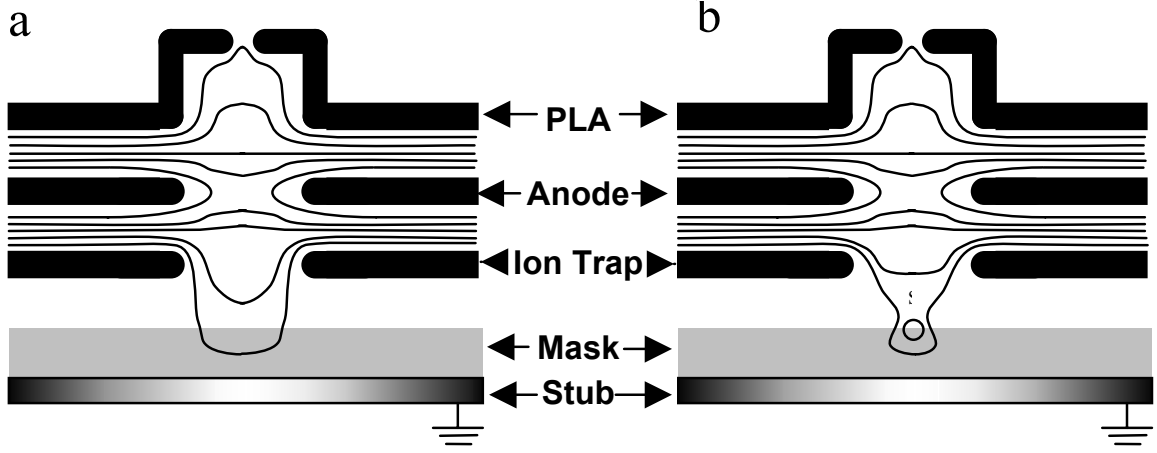

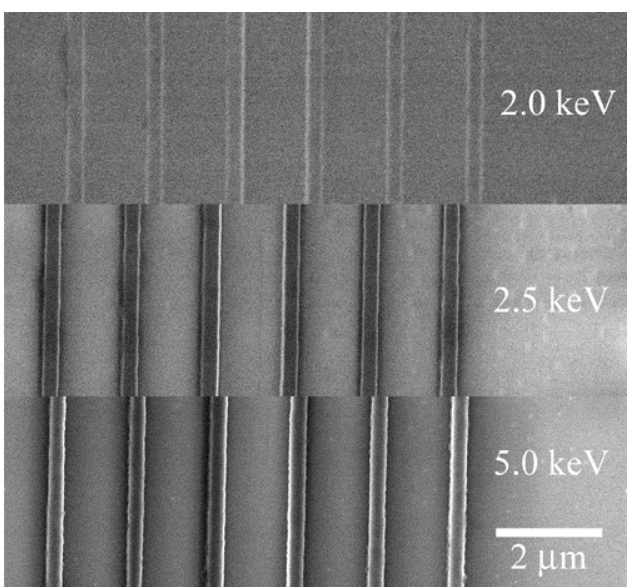

Figure 2. Low vacuum images of mask at $2.0,2.5$, and $5.0 \mathrm{keV}$. Contrast digitally enhanced for $2.0 \mathrm{keV}$ image.

Figure 3. Electrode geometry and electrostatic field structure for low vacuum Helix detector. (a) Field structure for uncharged specimen. (b) Field structure for positively charged specimen. $\mathrm{S}=$ field saddle point. 